\title{
Vascular Features of Nail Psoriasis Using Dynamic Optical Coherence Tomography
}

\author{
Adam S. Aldahan ${ }^{a}$ Lucy L. Chen ${ }^{a}$ Raymond M. Fertig ${ }^{a}$ Jon Holmes ${ }^{b}$ \\ Vidhi V.Shah ${ }^{a}$ Stephanie Mlacker ${ }^{a}$ Vincent M. Hsu ${ }^{a}$ Keyvan Nouri ${ }^{a}$ \\ Antonella Tostia \\ a Department of Dermatology and Cutaneous Surgery, University of Miami Miller School of Medicine, \\ Miami, Fla., USA; ${ }^{b}$ Michelson Diagnostics Ltd, Maidstone, UK
}

\section{Key Words}

Nail psoriasis · Nails · Psoriasis · Optical imaging · Vascular pattern - Optical coherence tomography

\begin{abstract}
Background: Nail psoriasis is a painful and disfiguring nail disease that often leads to invasive biopsies. Dermoscopy of the hyponychium can be useful in the diagnosis showing twisted coiled vessels. Structural features of nail psoriasis have been described with optical coherence tomography (OCT). Objectives: To investigate vascular features of nail psoriasis using dynamic OCT. Methods: This was an observational, prospective, controlled study in which psoriasis patients with psoriatic nail changes and healthy control patients underwent OCT imaging of the distal nail plate and proximal nail fold. Vertical and horizontal OCT images were analyzed to describe structural and vascular features and to quantify blood flow at depth. Results: Sixteen psoriatic nails and 16 control nails were included. Psoriatic nails had significantly increased blood flow in the proximal nail fold at depths of $0.72 \mathrm{~mm}(p=0.035)$ and $0.76 \mathrm{~mm}(p=0.027)$. Nail thickness was significantly greater in psoriatic nails compared to control nails $(p=0.0016)$. Compared to control nails, psoriatic nails had dilated, disorganized blood vessels
\end{abstract}

superficially in the proximal nail fold. Limitations: The main limitation of our study is the relatively small sample size. Conclusions: OCT can identify structural and vascular features specific to nail psoriasis.

(c) 2016 S. Karger AG, Basel

\section{Introduction}

Psoriasis is an immune-mediated disease that affects $2 \%$ of the North American and European population [1]. Nails are affected in up to $78 \%$ of patients with psoriasis and $70-80 \%$ of those with psoriatic arthritis $[1,2]$. Nail psoriasis is a painful, debilitating, and embarrassing disease that is often advanced at the time of diagnosis [3]. Psoriasis limited to the nails is not uncommon, and diagnosis can be difficult as the nail abnormalities may not be specific, as for instance, in patients presenting with onycholysis or subungual hyperkeratosis, which can be symptoms of many other nail disorders. Dilated tortuous vessels seen in the hyponychium on dermoscopy can indicate correct diagnosis $[4,5]$. The invasiveness and pain associated with nail biopsies limits their use in many cases.

Noninvasive techniques such as optical coherence tomography (OCT) and high-frequencyultrasound (HFUS)

\section{KARGER}

E-Mail karger@karger.com www.karger.com/sad (c) 2016 S. Karger AG, Basel

2296-9195/16/0024-0102\$39.50/0
Adam S. Aldahan, BS

1475 NW 12th Ave., Suite 2175

Miami, FL 33136 (USA)

E-Mail aaldahan@med.miami.edu 
may help establish a definitive diagnosis of psoriasis as well as monitor therapeutic response to treatment [6]. These particular modalities facilitate viewing of the epidermis and dermis, providing greater detail than more basic imaging techniques such as dermoscopy and videocapillaroscopy. Recent studies have shown OCT to have considerable diagnostic value in nail disease, advocating OCT as a superior modality to ultrasound due to higher resolution imaging $[7,8]$.

OCT provides the clinician with live, cross-sectional gray-scale images of tissue microarchitecture that have been shown to correlate well with histologic examination [9]. Recently, 'Dynamic OCT' has been developed, which detects particle movement such as blood flow, providing the ability to map blood vessel architecture to determine vascular patterns specific to disease processes $[10,11]$. Using Dynamic OCT, we are now able to describe structural and vascular features consistent with and specific to nail psoriasis.

\section{Patients and Methods}

\section{Study Population}

Patients with a clinical diagnosis of psoriasis and psoriatic nail changes were included in our study. All patients were recruited from December 2015 to April 2016 at the Outpatient Dermatology Clinic at the University of Miami, Miami, Fla., USA. Male and female patients of all ethnicities and Fitzpatrick skin types were eligible. Inclusion criteria were patients with skin psoriasis and clinical evidence of typical psoriatic changes in at least one nail $[1,2]$. Both treated patients and treatment-naive patients were eligible. We also recruited healthy control patients without psoriasis and without any evidence of nail disease for objective comparison.

Psoriatic nails were selected to undergo OCT imaging, as well as an equal number of nonpsoriatic nails in healthy patients. Clinical photographs and dermatoscopic images were obtained under standardized conditions in all nails selected for imaging. Informed consent was obtained from all participants prior to enrollment. This study was approved by the University of Miami Institutional Review Board and was conducted in accordance with the ethical standards of the Human Subject Research Office at the University of Miami.

\section{Optical Coherence Tomography}

Nails were imaged using the VivoSight Rx OCT device (Michelson Diagnostics Ltd, Maidstone, UK). This device provides a visual field of $6 \mathrm{~mm}$, a depth of $2 \mathrm{~mm}$, and a spatial resolution of $7.5 \mu \mathrm{m}$ in all axes. Light reflectance is detected by the device and converted into three-dimensional gray-scale images that allow visualization in horizontal and vertical planes. Because of the natural contour of skin and nail surfaces, we employed the 'Contoured En Face' mode of the VivoSight to visualize the en face, or horizontal, OCT image of all points with the same depth below the tissue surface. The dynamic OCT function detects motion of any part of the tissue, such as may be caused by blood cells flowing in vessels, by measuring the change in OCT signal intensity between two rapidly sequentially acquired OCT scans, and then displays red pixels with brightness corresponding to motion signal intensity. At present, the precise correspondence between dynamic OCT intensity and blood flow velocity is not known, but it has been shown that the dynamic OCT signal correlates well with changes in blood flow magnitude [12].

We scanned each nail at the distal nail plate and proximal nail fold. Cross-sectional and contoured en face images were obtained in each scan for qualitative visual comparison. We also calculated quantitative data such as linear measurements and dynamic blood flow data.

\section{Analysis of Data}

OCT images were analyzed without any digital enhancements to ensure accurate and unbiased comparisons. Data were compiled and analyzed using Microsoft Excel (2011). Independent t tests were employed to determine differences between two groups. All $\mathrm{p}$ values correspond to one-sided t tests with an alpha level of 0.05 .

Blood flow data of each proximal nail fold scan were extracted from the OCT device using the Blood Flow at Depth software program. This program first divides the OCT data into horizontal contoured sections at $0.04-\mathrm{mm}$ increments in depth below the tissue surface. It then outputs the total summed dynamic OCT signal at each depth and also partitions the signal into discrete levels of dynamic intensity. Dynamic intensities range from 2.5 to $97.5 \%$, with lower intensities constituting primarily noise and higher intensities more specific for blood flow. To reduce the noise bias, we analyzed only the dynamic OCT data at $97.5 \%$ dynamic intensity to represent blood flow. At very shallow depths corresponding to the epidermis, the dynamic intensity is normally very low. It then rises at depths corresponding to the papillary loops and then the superficial plexus. However, at greater depths corresponding to the mid- and deep dermis, the dynamic OCT signal falls due to limited penetration depth of the OCT imaging technique. To calculate the depth at which dynamic signal begins to drop off, we graphed the datasets of each group and calculated the inflection point of each graph using a 4th order polynomial regression model. Of the two graphs, the inflection point that was found at the more superficial level was used as our maximum evaluable depth.

\section{Results}

A total of 16 psoriatic nails in 7 patients (age 19-66 years) were imaged using OCT. Additionally, 16 control nails in 7 healthy patients (age 21-80 years) were imaged for comparison. Five psoriasis patients had been treated prior to imaging; 2 were treatment naïve. All subjects underwent OCT imaging by the same investigator (A.S.A.). All subjects had Fitzpatrick skin types between I and IV.

\section{Structural Features of Psoriatic Nails}

The clinical features of nail psoriasis were identified in our OCT images (fig. 1a-c). The distal psoriatic nails have dystrophic, wavy, layered nail plates that contain parallel 

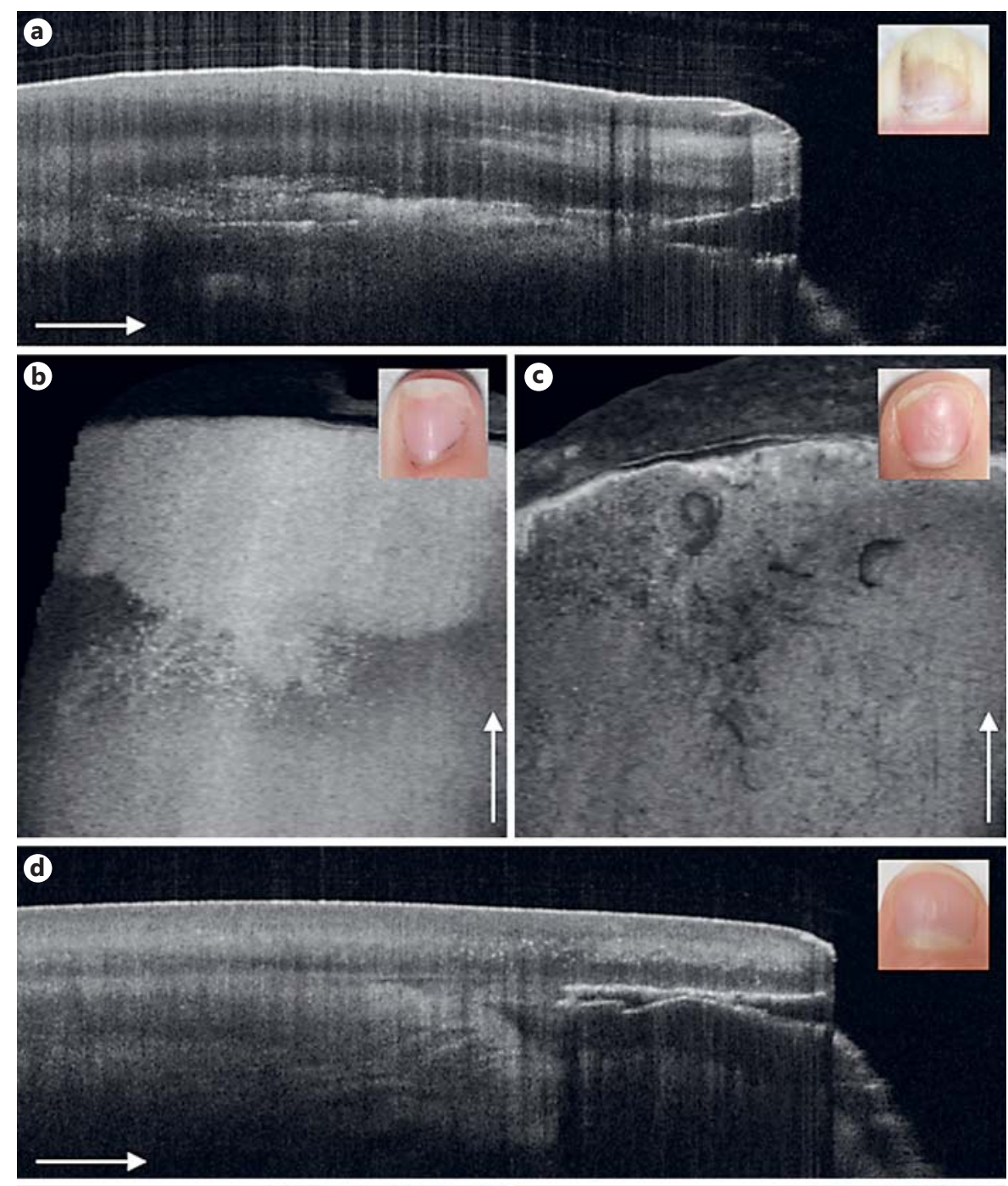

Fig. 1. Structural features of nail psoriasis. Arrows point in the direction of the distal nail. a Cross-sectional view of a distal psoriatic nail illustrating a wavy nail plate with white streaks and significant onycholysis overlying a highly reflective and irregular nail bed with white tufts. b En face view of a distal psoriatic nail illustrating pronounced leukonychia and extensive speckling (depth $0.16 \mathrm{~mm}$ ). c En face view of a distal psoriatic nail illustrating pitting (depth $0.30 \mathrm{~mm}$ ). $\mathbf{d}$ Cross-sectional view of a distal healthy nail illustrating a smooth nail surface, normal distribution of white speckles, and a linear regular nail bed proximal to the hyponychium. En face views of the same healthy nail at $0.16 \mathrm{~mm}(\mathbf{e})$ and $0.30 \mathrm{~mm}(\mathbf{f})$ in depth.
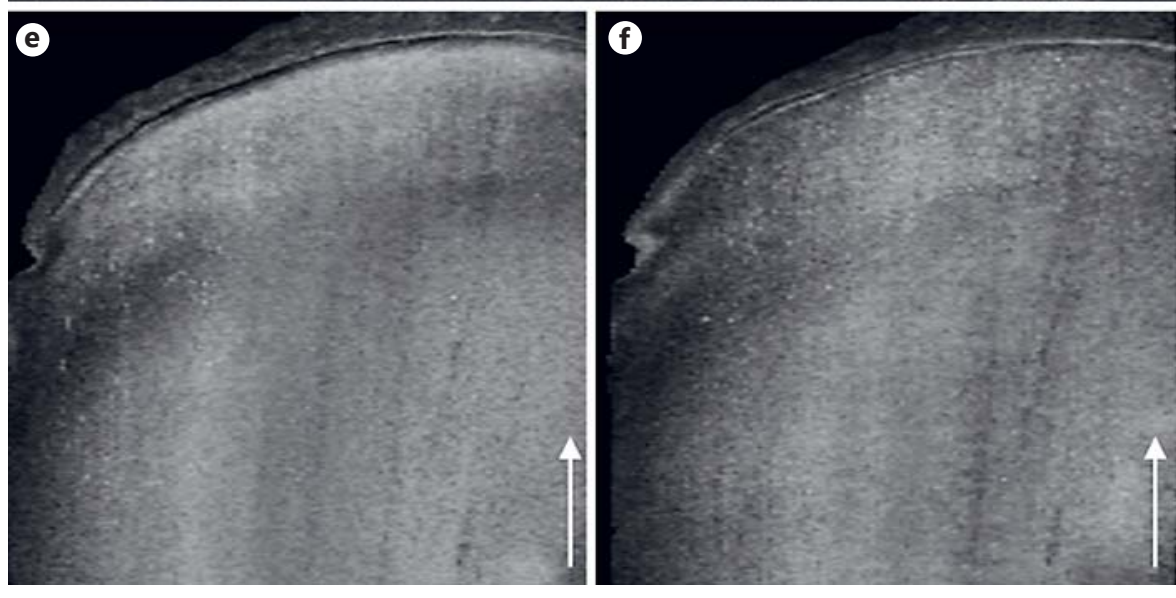
Fig. 2. Vascular features of nail psoriasis in the proximal nail fold. Arrows point in the direction of the distal nail. Note that the vertical red streaks visible in the cross-sectional view are shadow artifacts of the dynamic OCT technique. Colors refer to the online version only. a En face view of a psoriatic nail illustrating dilated vessels in a haphazard orientation (depth $0.6 \mathrm{~mm}$ ). b Cross-sectional view of the same psoriatic nail illustrating increased density of blood vessels protruding superficially. c En face view of a healthy nail (depth $0.6 \mathrm{~mm}$ ). d Cross-sectional view of the same healthy nail.
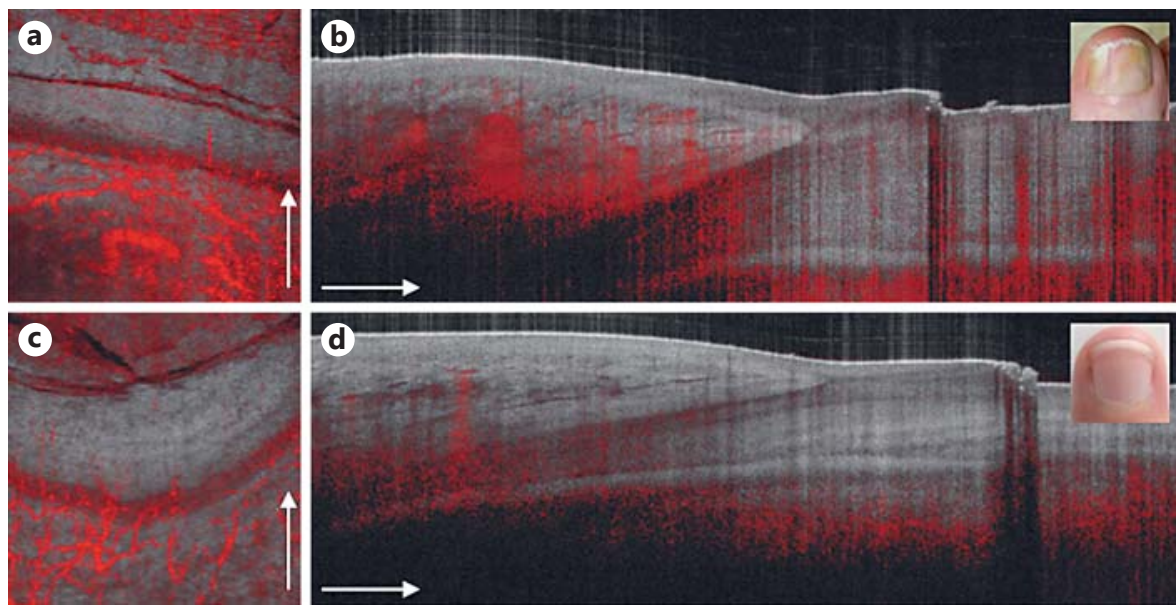

white streaks (fig. 1a). The nail surface is rough and irregular with superficial fissuring. The white streaks within the nail plate correlate clinically as leukonychia and are seen predominantly in the mid-layer of the nail (fig. 1a, b) [13]. The white speckled pattern in the nail plate can also be seen in controls but seems to be more abundant in the psoriatic nail (fig. 1b). Pitting is also clearly visualized as black ringed shapes (fig. 1c) that can be traced to a measurable depth using OCT. Cross-sectional views display pitting as a focal dark irregularity with underlying shadowing. The psoriatic nails were significantly thicker than healthy nails. The average nail thickness in psoriatic nails was $0.65 \mathrm{~mm}$ compared to $0.47 \mathrm{~mm}$ in the control nails ( $\mathrm{p}=0.0016,95 \%$ CI $0.0622,0.2958$ ).

In addition to nail plate findings, we were able to appreciate abnormalities within the nail bed in the areas of onycholysis consisting of subungual ridges alongside highly reflective white wispy sheets and dense white specks. The deep nail bed also contains predominant black empty areas surrounding highly reflective tufts that resemble cotton balls or cloud-like structures. These nail bed findings are unique to psoriatic nails and are not seen in the healthy controls, where the nail bed contains homogeneously reflective tissue with a linear superficial border proximal to the hyponychium (fig. 1d). The OCT signal begins to diminish below the nail bed, seen as gradually uniform signal darkening.

\section{Structural and Vascular Features of the Proximal Nail Fold}

Using cross-sectional and en face OCT views standardized at $0.60 \mathrm{~mm}$ in depth, we were able to depict dilated vessels with a haphazard architecture in the psoriatic nails (fig. 2a). Additionally, increased dynamic signal was dif- fusely seen deep to the proximal nail plate itself despite the increased nail thickness. The cross-sectional view shows a dense arrangement of blood vessels extending superficially (fig. 2b). Structurally, the skin surface is ragged and indurated, meeting the proximal nail at a steep angle.

Conversely, healthy nails contain thin blood vessels with an organized reticular pattern that is visualized with the en face view (fig. 2c). No defined vascular architecture is seen distal to the cuticle under the nail plate, and the overall dynamic signal is reduced. The vessels are less striking in the cross-sectional view and do not extend as superficially (fig. $2 \mathrm{~d}$ ). The skin overlying the proximal nail fold is also more linear and meets the nail plate at a shallow angle.

Quantification of the blood flow in both the psoriatic and healthy nails on OCT using the dynamic signal intensity of $97.5 \%$ allowed us to establish the depth range over which the OCT device was able to completely detect dynamic signal. We determined the maximum evaluable depth in each group by graphically plotting the average signal at each depth and calculating the inflection point, which indicates a gradual decline in signal detection. This value was $0.84 \mathrm{~mm}$ in the psoriasis group and $0.76 \mathrm{~mm}$ in the control group. To avoid any biases due to signal dropoff, we used the lesser of the two values and determined our maximum evaluable depth to be $0.76 \mathrm{~mm}$. Figure 3 illustrates a graphical comparison of blood flow signal with increasing depth in each group. Complete blood flow signal results of the psoriatic nails and healthy control nails are presented in table 1 . Psoriatic nails had significantly increased blood flow signals at 0.72 and 0.76 $\mathrm{mm}$. Signals between 0.40 and $0.72 \mathrm{~mm}$ deep trended toward an increase in the psoriatic nail group but did not reach significance. 


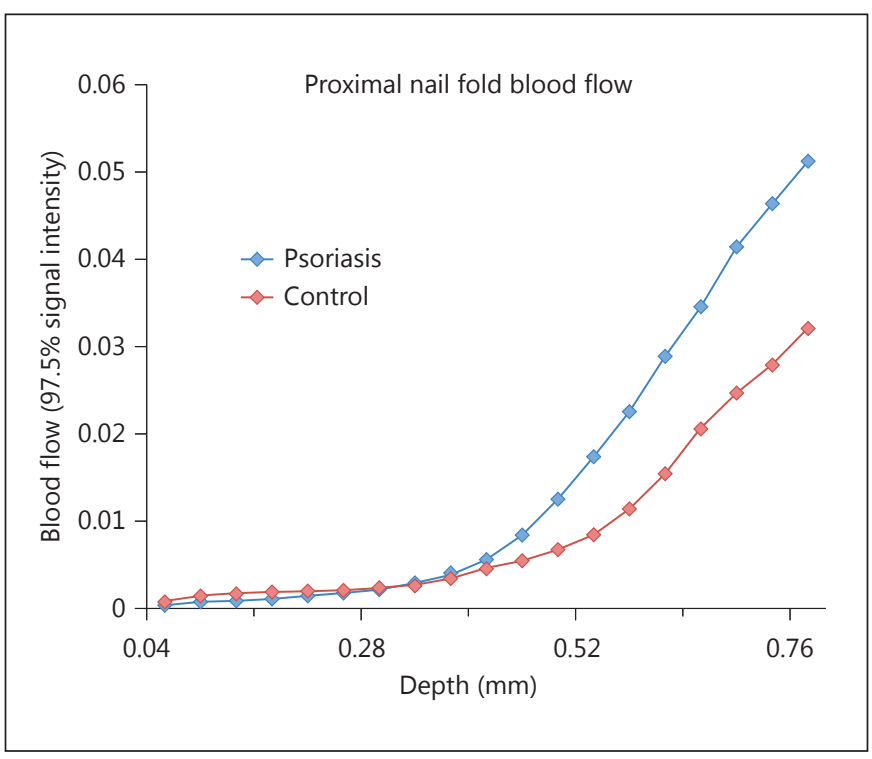

Fig. 3. Quantitative blood flow measurements of proximal nail folds in psoriatic nails and healthy control nails.

Table 1. Properties of psoriatic nails on OCT

\begin{tabular}{clll}
\hline & $\begin{array}{l}\text { Psoriatic nails } \\
(\mathrm{n}=13)\end{array}$ & $\begin{array}{l}\text { Healthy nails } \\
(\mathrm{n}=13)\end{array}$ & $\mathrm{p}$ value \\
\hline Nail thickness, mm $^{\text {PNF dynamic signal }}{ }^{\mathrm{b}}$ & $0.65(0.209)^{\mathrm{a}}$ & $0.47(0.077)^{\mathrm{a}}$ & $0.0016^{*}$ \\
$0.48 \mathrm{~mm}$ & 0.013 & 0.007 & 0.129 \\
$0.52 \mathrm{~mm}$ & 0.017 & 0.008 & 0.090 \\
$0.56 \mathrm{~mm}$ & 0.023 & 0.011 & 0.080 \\
$0.60 \mathrm{~mm}$ & 0.029 & 0.015 & 0.066 \\
$0.64 \mathrm{~mm}$ & 0.035 & 0.021 & 0.071 \\
$0.68 \mathrm{~mm}$ & 0.041 & 0.025 & 0.053 \\
$0.72 \mathrm{~mm}$ & 0.046 & 0.028 & $0.035^{*}$ \\
$0.76 \mathrm{~mm}$ & 0.051 & 0.032 & $0.027^{*}$ \\
& & &
\end{tabular}

PNF $=$ Proximal nail fold. ${ }^{\text {a }}$ Values are shown as mean (SD).

b Values represent total expression at $97.5 \%$ dynamic signal intensity.

\section{Discussion}

Noninvasive imaging has an important role in the diagnosis and treatment monitoring of nail disease. HFUS, reflectance confocal microscopy (RCM), and OCT have been studied for noninvasive in vivo imaging. Each of these modalities varies in depth of penetration, resolution, and visual representation. HFUS provides the deepest penetration depth, ranging from 1 to $12 \mathrm{~mm}$ depending on the frequency utilized (typically $100-20 \mathrm{MHz}$ ), which allows for the visualization of the epidermis and deep dermis [6]. OCT and RCM are relatively limited in depth penetration compared to HFUS. OCT has a penetration depth of up to $2 \mathrm{~mm}$, while RCM achieves the least penetration depth of $250-350 \mu \mathrm{m}$, equivalent to the level of the papillary dermis and upper reticular dermis [14].

In terms of resolution, however, HFUS is the most inferior, with resolution ranging from 32 to $200 \mu \mathrm{m}$ [6]. RCM affords the highest resolution with a lateral resolution of approximately $0.5-1.0 \mu \mathrm{m}$. At a resolution of $1 \mu \mathrm{m}$, RCM allows visualization at the cellular and nuclear level [6]. Lateral resolution for OCT ranges from 1 to $20 \mu \mathrm{m}$, approaching that of RCM. Recently, high-definition OCT scanners have been developed that provide lateral resolution of $1-3 \mu \mathrm{m}[6]$.

As for visual representation, HFUS captures images in the vertical plane while RCM captures images in the horizontal plane [15]. In contrast, OCT has the advantage of capturing images in both the horizontal and vertical planes. The main advantage of OCT, in addition to realtime imaging of tissue microstructure, is the capability of optical microangiography, which produces three-dimensional images of dynamic blood perfusion within the microcirculatory tissue beds. This allows for the creation of high-resolution vascular images without the use of any contrast agents to provide a detailed view of blood vessels in psoriatic plaques [6].

A major practical advantage to OCT over RCM is the speed of the procedure without the need for additional gel or medium at the skin surface. Recent studies have advocated that OCT is superior to ultrasound especially for nail imaging due to its higher resolution $[7,8]$. These studies revealed specific structural findings for certain nail diseases with OCT; however, the existing literature predates the dynamic feature of OCT, and therefore the previous studies drew no conclusions regarding vessel patterns. This is the first study that employs the dynamic feature of OCT to quantify blood flow and visualize vessel architecture in nail disease.

The layered appearance we saw in the nail plate was previously described by Sattler et al. [16]. They used OCT to determine healthy nail plate thickness to range from about 0.45 to $0.50 \mathrm{~mm}$. These findings were consistent with our results of healthy control patients, with nail thickness falling within this range. Abuzahra et al. [17] distinguished nail psoriasis from onychomycosis. Unlike nail psoriasis, which consists of homogeneous scattering within the nail plate, onychomycosis contains a high scattering area surrounded by a low scattering area, indicating the presence of fungus. 
Nail psoriasis can be a clinical mimicker to a host of other nail diseases. Dermoscopy literature has aided in describing findings specific to nail psoriasis including onycholysis with fine longitudinal striations, as well as an increased density of dilated, tortuous capillaries in the hyponychium $[4,5]$. Many of these specific findings were corroborated on OCT, such as the fine white striations in the distal nail plate. OCT may serve as an alternative diagnostic tool for patients who cannot tolerate painful, invasive nail biopsies, including children.

The structural and dynamic information gained by in vivo OCT imaging far exceeds that of dermoscopy and videocapillaroscopy. This additional information may be useful for diagnosis as well as treatment monitoring in psoriatic nails. In nail psoriasis specifically, we were able to identify and objectively quantify novel findings such as an increased vessel size, vessel density, and thickened epidermis in the proximal nail fold, previously only seen on histopathology [18]. Psoriasis presents histopathologically with dilated capillaries in the papillary dermis, which is at a depth well within the reach of dynamic OCT $[18,19]$. We were able to detect a significant increase in blood flow less than $1 \mathrm{~mm}$ deep in psoriatic proximal nail folds. The haphazard vessel architecture seen in the proximal nail fold was similarly seen deep to the nail bed in some subjects, which may correlate with nail severity. Although signal drop-off prevented us from quantifying the deeper blood flow, visual inspection revealed a consistent difference between the two groups.

The various structural features of nail psoriasis can present together in the same nail or separately in distinct nails. Furthermore, many patients only have one or a few affected nails. Our OCT findings of structural nail changes predominantly reflected clinical features, with some additional information regarding extent into the nail bed. OCT exposed abnormalities such as nail bed ridges with patchy white tufts, which is beyond the scope of clinical examination.

\section{Limitations}

The main limitation of our study is the relatively small sample size, although our specific findings were present throughout the patients with psoriasis. The hyponychium in particular is difficult to visualize with OCT due to the scattering of light by the distal nail plate. We were unable to clearly appreciate the characteristic tortuous hyponychial capillaries even after repositioning the hand piece several times from different perspectives. The thick psoriatic nails also hindered visualization of vascular architecture beneath the nail plate in most subjects. One minor practical drawback to OCT is the rather steep learning curve in terms of maneuvering the device on nails. Operating the OCT device is userdependent, and maintaining steady hands to reduce signal noise takes time and improves with practice. Variations in skin pressure may also affect the dynamic data due to vessel compression. Additionally, other factors affecting vessel size such as peripheral vascular disease and drug use could have potentially confounded our results. Nevertheless, the high-resolution information generated by OCT without the invasiveness of biopsies makes it a valuable tool for the evaluation of underlying nail disease.

\section{Acknowledgments}

We thank Michelson Diagnostics Ltd, Maidstone, UK, for providing the OCT device.

\section{Statement of Ethics}

Informed consent was obtained from all participants prior to enrollment. This study was approved by the University of Miami Institutional Review Board and was conducted in accordance with the ethical standards of the Human Subject Research Office at the University of Miami.

\section{Disclosure Statement}

Jon Holmes is an employee of Michelson Diagnostics Ltd. All other authors declare no conflicts of interest. The VivoSight OCT device was loaned by Michelson Diagnostics Ltd.

References

1 Boehncke WH, Schon MP: Psoriasis. Lancet 2015;386:983-994.

2 Dogra A, Arora AK: Nail psoriasis: the journey so far. Indian J Dermatol 2014;59:319333.

3 Ortonne JP, Baran R, Corvest M, Schmitt C, Voisard JJ, Taieb C: Development and validation of nail psoriasis quality of life scale (NPQ10). J Eur Acad Dermatol Venereol 2010;24:22-27.

4 Iorizzo M, Dahdah M, Vincenzi C, Tosti A Videodermoscopy of the hyponychium in nail bed psoriasis. J Am Acad Dermatol 2008; 58:714-715.

5 Farias DC, Tosti A, Chiacchio ND, Hirata SH Dermoscopy in nail psoriasis (in Portuguese). An Bras Dermatol 2010;85:101-103. 
6 Lacarrubba F, Pellacani G, Gurgone S, Verzi AE, Micali G: Advances in non-invasive techniques as aids to the diagnosis and monitoring of therapeutic response in plaque psoriasis: a review. Int J Dermatol 2015;54:626-634.

7 Aydin SZ, Castillo-Gallego C, Ash ZR, Abignano $G$, Marzo-Ortega $H$, Wittmann M, Del Galdo F, McGonagle D: Potential use of optical coherence tomography and high-frequency ultrasound for the assessment of nail disease in psoriasis and psoriatic arthritis. Dermatology 2013;227:45-51.

8 Aydin SZ, Ash Z, Del Galdo F, Marzo-Ortega $\mathrm{H}$, Wakefield RJ, Emery P, McGonagle D: Optical coherence tomography: a new tool to assess nail disease in psoriasis? Dermatology 2011;222:311-313.

9 Schuh S, Kaestle R, Sattler EC, Welzel J: Optical coherence tomography of actinic keratoses and basal cell carcinomas - differentiation by quantification of signal intensity and layer thickness. J Eur Acad Dermatol Venereol 2016;30:1321-1326.
10 Mariampillai A, Standish BA, Moriyama EH, Khurana M, Munce NR, Leung MK, Jiang J, Cable A, Wilson BC, Vitkin IA, Yang VX: Speckle variance detection of microvasculature using swept-source optical coherence tomography. Opt Lett 2008;33:1530-1532.

11 Ulrich M, Themstrup L, de Carvalho N, Manfredi M, Grana C, Ciardo S, Kastle R, Holmes J, Whitehead R, Jemec GB, Pellacani G, Welzel J: Dynamic optical coherence tomography in dermatology. Dermatology 2016;232:298311.

12 Themstrup L, Ciardo S, Manfredi M, Ulrich M, Pellacani G, Welzel J, Jemec GB: In vivo, micro-morphological vascular changes induced by topical brimonidine studied by dynamic optical coherence tomography. J Eur Acad Dermatol Venereol 2016;30:974-979.

13 Mogensen M, Thomsen JB, Skovgaard LT, Jemec GB: Nail thickness measurements using optical coherence tomography and 20MHz ultrasonography. Br J Dermatol 2007; 157:894-900.

14 Ulrich $\mathrm{M}$, von Braunmuehl $\mathrm{T}$, Kurzen $\mathrm{H}$, Dirschka T, Kellner C, Sattler E, Berking C, Welzel J, Reinhold U: The sensitivity and specificity of optical coherence tomography for the assisted diagnosis of nonpigmented basal cell carcinoma: an observational study. Br J Dermatol 2015;173:428-435.
15 Basaran YK, Gurel MS, Erdemir AT, Turan E, Yurt N, Bagci IS: Evaluation of the response to treatment of psoriasis vulgaris with reflectance confocal microscopy. Skin Res Technol 2015;21:18-24.

16 Sattler E, Kaestle R, Rothmund G, Welzel J: Confocal laser scanning microscopy, optical coherence tomography and transonychial water loss for in vivo investigation of nails. $\mathrm{Br}$ J Dermatol 2012;166:740-746.

17 Abuzahra F, Spoler F, Forst M, Brans R, Erdmann S, Merk HF, Obrigkeit DH: Pilot study: optical coherence tomography as a non-invasive diagnostic perspective for real time visualisation of onychomycosis. Mycoses 2010;53: 334-339.

18 De Rosa G, Mignogna C: The histopathology of psoriasis. Reumatismo 2007;59(suppl 1): 46-48.

19 Robertson K, Rees JL: Variation in epidermal morphology in human skin at different body sites as measured by reflectance confocal microscopy. Acta Derm Venereol 2010;90:368373 . 